\title{
BIBLIOGRAPHY
}

1. H. M. Elliott, On approximation to functions satisfying a generalized continuity condition, Trans. Amer. Math. Soc. vol. 71 (1951) pp. 1-23.

2. W. E. Sewell, Continuity and degree of approximation by rational functions, Revista de Ciencias vol. 41 (1939) pp. 435-451.

3. J. L. Walsh, Interpolation and approximation by rational functions in the complex domain, Amer. Math. Soc. Colloquium Publications, vol. 20, New York, 1935.

4. J. L. Walsh and W. E. Sewell, Sufficient conditions for various degrees of approximation by polynomials, Duke Math. J. vol. 6 (1940) pp. 658-705.

WASHINGTON UNIVERSITY

\section{A CONVEX METRIC WITH UNIQUE SEGMENTS}

R. H. BING

1. Introduction. If $D(x, y)$ is a convex metric for a continuous curve $M$ (compact, locally connected, metric continuum), then for each pair of points $p, q$ of $M$ there is an arc $p q$ in $M$ from $p$ to $q$ such that $p q$ is isometric to a straight line interval. We shall call it a segment.

Each continuous curve has a convex metric $[1 ; 3 ; 5 ; 6 ; 2]$. However, if $D(x, y)$ is a convex metric for $M$, there may be two segments from $p$ to $q$. If $M=S_{2}$ is the surface of a sphere, $D(x, y)$ is the size of the central angle subtended by $x$ and $y$, and $p, q$ are diametrically opposite points, then there are many segments from $p$ to $q$. In fact, we show in $\$ 4$ that if $D(x, y)$ is any convex metric whatsoever for $S_{2}$, each point of $S_{2}$ belongs to a pair of points which are not joined by a unique segment.

There is a dense subset $W$ of $S_{2}$ such that no two points of $W$ are diametrically opposite. If $D(x, y)$ is the previously mentioned convex metric for $S_{2}$, then each pair of points of $W$ are joined by one and only one segment. We shall show that for any continuous curve there is such a dense subset and such a convex metric.

Theorem. Each continuous curve $M$ has a dense subset $W$ and a convex metric $D(x, y)$ such that each pair of points of $W$ belongs to a unique segment.

We shall prove this result in much the same manner that it was shown that any continuous curve can be convexified. The metric is

Presented to the Society September 7, 1951; received by the editors March 21, 1952. 
an adaption and modification of that described in Theorem 4 of [5]. We consider a decreasing sequence of core partitionings of $M$, assign sizes to the pieces of the partitionings, and define the distance between two points in terms of the sizes of the chains joining them. The convex metric $D(x, y)$ is described in $\$ 2$ and the dense set $W$ is given in $\$ 3$.

We recall the following definitions. A partitioning of $M$ is a finite collection of mutually exclusive connected open subsets of $M$ whose sum is dense in $M$. A sequence of partitionings is decreasing if for each integer $i, G_{i+1}$ is a refinement of $G_{i}$ (each element of $G_{i+1}$ lies in an element of $G_{i}$ ) and the mesh of $G_{i}$ (maximum of diameters of elements of $G_{i}$ ) approaches 0 as $i$ increases without limit.

A partitioning is regular if each of its pieces is the interior of its closure. If the partitioning $H$ is a refinement of the regular partitioning $K$, the elements of $H$ which have a boundary point in common with a boundary of an element of $K$ are called border elements. Other elements are interior elements.

We call $H$ a core refinement of $K$ if each is regular, $H$ is a refinement of $K$, each border element of $H$ is adjacent to an interior element, and the sum of the interior elements of $H$ in each element of $K$ has a connected closure.

2. A convex metric for $M$. In this section we describe a convex metric $D(x, y)$ for $M$. We find in $\$ 3$ that there is a dense subset $W$ of $M$ such that under $D(x, y)$, each pair of points of $W$ belong to a unique segment.

Description of convex metric $D(x, y)$. It was shown in [3] that $M$ has a decreasing sequence $G_{1}, G_{2}, \cdots$ of regular partitionings such that each is a core refinement of the preceding and each element of $G_{i+1}$ is of diameter less than one-third the distance between any two nonadjacent elements of $G_{i}$.

Suppose the elements of the partitioning $G_{i}$ are ordered $g_{i 1}, g_{i 2}, \cdots$, $g_{i n_{i}}$. We assign sizes to the elements of $G_{i}$ as follows. The element $g_{1 j}$ of $G_{1}$ is assigned a size $1 / 4^{j}$. Each interior element $g_{2 j}$ of $G_{2}$ is assigned a size $1 / 4^{j+n_{1}}$ where $n_{1}$ is the number of elements in $G_{1}$. If $g_{2 j}$ is a border element of $G_{2}$, its size is one-half the size of the element of $G_{1}$ containing it plus $1 / 4^{i+n_{1}}$. In general, the size of $g_{i j}$ is the first or second of the following expressions according as $g_{i j}$ is an interior of a border element of $G_{i}$.

$$
\text { Size }\left(g_{i j}\right)=\left\{\begin{array}{l}
1 / 4^{i+n_{1}+n_{2}+\cdots+n_{j-1}} \text { or } \\
1 / 4^{j+n_{1}+n_{2}+\cdots+n_{j-1}}+1 / 2 \text { size of element of } \\
G_{i-1} \text { containing } g_{i j} .
\end{array}\right.
$$


Had we not been interested in building a convex metric with unique segments, we would have made the following simplification in the definition of the sizes of the elements of $G_{i}$ : each interior element of $G_{i}$ would be given a size $1 /\left(2^{i} \cdot n_{i}\right)$ and each border element of $G_{i}$ would be given a size of one-half the size of the element of $G_{i-1}$ containing it.

If $g$ is an element of $G_{i}$, we denote its size by $S_{i}(g)$. If $p$ is a point of $g$, we shall also denote $S_{i}(g)$ by $S_{i}(p)$. If $G$ is a collection of elements of $G_{i}$, the sum of the sizes of the elements of $G$ is denoted by $S_{i}(G)$.

Suppose $K$ is a continuum which is the closure of the sum of a subcollection of $G_{i}$. The sum of the sizes of the elements of $G_{i}$ in $K$ is called the $i$ th size of $K$ and is denoted by $S_{i}(K)$.

If $p$ and $q$ are two points of $M$, we define $E_{i}(p, q)$ to be the minimum of all numbers $S_{i}(K)$ where $K$ is a continuum with $i$ th size which contains $p+q$. We may regard $E_{i}(p, q)$ as the $i$ th approximation to the distance between $p$ and $q$. Then $D(p, q)=\lim E_{i}(p, q)$.

Existence of $\lim E_{i}(p, q)$. Suppose $K$ is a continuum containing $p+q$ and having an $i$ th size of $E_{i}(p, q)$. It is the closure of the sum of a chain of elements of $G_{i}$ such that $p$ and $q$ lie in the closures of the end links of this chain. There is a continuum $K^{\prime}$ in $K$ containing $p+q$ such that $K^{\prime}$ has an $(i+1)$ st size but does not contain three border elements of $G_{i+1}$ in the same element of $G_{i}$. Then $S_{i+1}\left(K^{\prime}\right)$ $\leqq S_{i}(K)+1 / 4^{1+n_{1}+\cdots+n_{i}}+\cdots+1 / 4^{n_{1+n_{2}+\cdots+n_{i+1}}}$. Therefore

(1) $E_{i+1}(p, q)+1 /\left(3 \cdot 4^{n_{1}+n_{2}+\cdots+n_{i+1}}\right) \leqq E_{i}(p, q)+1 /\left(3 \cdot 4^{n_{1}+n_{2}+\cdots+n_{i}}\right)$.

Now inequality (1) implies that $\left\{E_{i}(p, q)+1 /\left(3 \cdot 4^{n_{1}+n_{2}+\cdots+n_{i}}\right)\right\}$ is a monotone nonincreasing sequence of positive numbers. Hence $\lim E_{i}(p, q)$ exists.

In fact, $E_{i}(p, q)$ converges to $D(x, y)$ uniformly since it can be shown that $\left|D(p, q)-E_{i}(p, q)\right|<2 / 2^{i}$.

That $D(x, y)$ is a convex metric for $M$ follows by an argument similar to that used in [3] to show that a continuous curve has a convex metric.

3. Unique segments in $M$. In this section we show that if $M$ is given the convex metric $D(x, y)$ described in the last section, there is a dense subset $W$ of $M$ such that each pair of points of $W$ belongs to a unique segment.

Description of set $W$. Let $G_{1}, G_{2}, \cdots$ be the decreasing sequence of core partitionings of $M$ described in the last section. Let $C_{i}$ be the sum of the core elements of $G_{i}$ and $W_{i}$ be the intersection of $C_{i}, C_{i+1}$, 
$C_{i+2}, \cdots$. Then $W_{i}$ is a closed set that intersects each element of $G_{i-1}$. We shall show that each pair of points of $W=W_{2}+W_{3}+\cdots$ belongs to a unique segment in $M$.

$A$ unique shortest ith chain from $p$ to $q$. If $g$ and $g^{\prime}$ are two elements of $G_{i}$, the denominators of $S_{i}(g)$ and $S_{i}\left(g^{\prime}\right)$ will lie between $4^{1+n_{1}+\cdots+n_{i-1}}$ and $4^{n_{1}+n_{8}+\cdots+n_{i}}$ inclusive but they will not be equal. Therefore, if $F_{1}$ and $F_{2}$ are two subcollections of $G_{i}$,

$$
\left|S_{i}\left(F_{1}\right)-S_{i}\left(F_{2}\right)\right| \geqq 1 / 4^{n_{1}+n_{2}+\cdots+n_{i}} .
$$

Hence, for each pair of points $p, q$ there is a unique continuum $K$ containing $p+q$ such that $S_{i}(K)=E_{i}(p, q)$.

Finding a continuum $H$ of ith size near a continuum $K$ of $(i+1)$ st size. If $K$ is a continuum with an $(i+1)$ st size and $g_{1}, g_{2}$ are two elements of $G_{i}$ intersecting $K$, there is a continuum $H$ with an $i$ th size such that $H$ contains $g_{1}+g_{2}, H$ is adjacent to each element of $G_{i}$ that is adjacent to $K$, and

$$
S_{i}(H)<S_{i+1}(K)+S_{i}\left(g_{1}\right) / 2+S_{i}\left(g_{2}\right) / 2 .
$$

We obtain the continuum $H$ in the following fashion. Let $G^{\prime}$ be the collection of border elements of $G_{i+1}$ that lie in $K$ and $G^{\prime \prime}$ be the collection of all elements $g$ of $G^{\prime}$ such that $g$ lies in neither $g_{1}, g_{2}$, nor any element of $G_{i}$ containing two elements of $G^{\prime}$. Then $G^{\prime \prime}$ is the sum of two mutually exclusive subcollections $G_{1}{ }^{\prime \prime}, G_{2}{ }^{\prime \prime}$ such that each element of $G^{\prime \prime}$ is adjacent to an element of $\left(G^{\prime}-G^{\prime \prime}\right)+G_{1}{ }^{\prime \prime}$ and also to an element of $\left(G^{\prime}-G^{\prime \prime}\right)+G_{2}^{\prime \prime}$. Let $G_{k}{ }^{\prime \prime}$ be the one of $G_{1}^{\prime \prime}, G_{2}^{\prime \prime}$ such that the sum of the $(i+1)$ st sizes of its elements is the smaller. Then $H$ is the closure of the sum of all elements $g$ of $G_{i}$ such that $g$ contains an element of $\left(G^{\prime}-G^{\prime \prime}\right)+G_{k}{ }^{\prime \prime}$.

Since each element of $G^{\prime}$ is adjacent to $H$ and the mesh of $G_{i+1}$ is less than the distance between nonadjacent elements of $G_{i}$, each element of $G_{i}$ that is adjacent to $K$ is also adjacent to $H$.

Let $F^{\prime}$ be the collection of elements of $G_{i}$ which contain an element of $G^{\prime}-G^{\prime \prime}$ and $F^{\prime \prime}$ be the collection of those that contain an element of $G_{k}^{\prime \prime}$. Since $S_{i}\left(F^{\prime \prime}\right)<S_{i+1}\left(G^{\prime \prime}\right)$ and $S_{i}\left(F^{\prime}\right)<S_{i+1}\left(G^{\prime}-G^{\prime \prime}\right)+S_{i}\left(g_{1}\right)$ $+S_{i}\left(g_{2}\right)$, inequality (3) follows.

Unless the collection of elements of $G_{i}$ that irreducibly cover the sum of the elements of $G_{1}{ }^{\prime \prime}$ is the same as those that irreducibly cover the sum of the elements of $G_{2}^{\prime \prime}$, it follows from inequality (2) that $S_{i}\left(F^{\prime \prime}\right)+1 /\left(2 \cdot 4^{n_{1}+n_{2}+\cdots+n_{i}}\right)<S_{i+1}\left(G^{\prime \prime}\right)$. Hence, unless $H$ contains $K$, we obtain the following strong form of inequality (3):

(4) $S_{i}(H)+1 /\left(2 \cdot 4^{n_{1}+n_{3}+\cdots+n_{i}}\right)<S_{i+1}(K)+S_{i}\left(g_{1}\right) / 2+S_{i}\left(g_{2}\right) / 2$. 
Shortest $(i+1)$ st chain lies in shortest ith chain. Suppose $p$ and $q$ are two points of $W_{i}$ and $H, K$ are the two continua such that each contains $p+q, S_{i}(H)=E_{i}(p, q)$, and $S_{i+1}(K)=E_{i+1}(p, q)$. We shall show that $K$ lies in $H$.

There is a continuum $K^{\prime}$ in $H$ containing $p+q$ such that $S_{i+1}\left(K^{\prime}\right)$ $\leqq S_{i}(H)-S_{i}(p) / 2-S_{i}(q) / 2+1 / 4^{1+n_{1}+\cdots+n_{i}}+\cdots+1 / 4^{n_{1}+n_{2}+\cdots+n_{i}+1}$. Therefore

$$
\begin{aligned}
S_{i+1}(K) \leqq & S_{i}(H)-S_{i}(p) / 2-S_{i}(q) / 2 \\
& +1 /\left(3 \cdot 4^{n_{1}+n_{2}+\cdots+n_{i}}\right)-1 /\left(3 \cdot 4^{n_{1}+n_{2}+\cdots+n_{i+1}}\right) .
\end{aligned}
$$

Now it follows from inequality (3) that there is a continuum $H^{\prime}$ containing $p+q$ such that $S_{i}\left(H^{\prime}\right)<S_{i+1}(K)+S_{i}(p) / 2+S_{i}(q) / 2$. This and inequality (5) implies that $S_{i}\left(H^{\prime}\right)-S_{i}(H)<1 / 4^{n_{1}+n_{2}+\cdots+n_{i}}$ and inequality (2) shows that $H^{\prime}=H$.

Unless $K$ lies in $H^{\prime}=H$, it follows from inequality (4) that $S_{i}(H)$ $+1 /\left(2 \cdot 4^{n_{1}+n_{2}+\cdots+n_{i}}\right)<S_{i+1}(K)+S_{i}(p) / 2+S_{i}(q) / 2$. This is impossible because of inequality (5). Therefore $K$ is a subset of $H$.

The unique segment. Suppose $p$ and $q$ are two points of $W$ and $K_{i}$ is the continuum containing $p+q$ such that $S_{i}\left(K_{i}\right)=E_{i}(p, q)$. If $m$ is an integer such that $p$ and $q$ belong to core elements of $G_{n}$ if $n \geqq m$, we find that $K_{m} \supset K_{m+1} \supset K_{m+2} \supset \ldots$. In fact, since the chain of closures of elements of $G_{m+i+1}$ whose sum is $K_{m+i+1}$ runs straight through the chain of closures of elements of $G_{m+i}$ whose sum is $K_{m+i}$ without doubling back and zigzagging, $K_{m} \cdot K_{m+1} \cdots$ is an $\operatorname{arc} p q$ from $p$ to $q$. We show that $p q$ is a unique segment from $p$ to $q$ by showing that any point not on $p q$ is not between $p$ and $q$.

Suppose $r$ is a point between $p$ and $q$ but not on $p q$. Let $H_{i}$ be the sum of the closures of all elements of $G_{i}$ adjacent to $K_{i}$. Then there is an integer $t$ greater than $m$ such that $r$ does not belong to $H_{t}$. Furthermore, for each positive number $\epsilon$ there is a positive integer $s$ and a continuum $R_{s}$ containing $p+r+q$ such that $S_{t+o}\left(R_{s}\right)<D(p, q)$ $+\epsilon$. We show that there is no such point $r$ by showing that there is no integer $s$ for $\epsilon=2 /\left(3 \cdot 4^{n_{1}+n_{2}+\cdots+n_{t}}\right)$.

The continuum $R_{s}$ is adjacent to $M-H_{t}$. By inequality (3) there is a continuum $R_{0-1}$ containing $p+q$ such that $R_{0-1}$ is adjacent to $M-H_{t}$ and

$$
\begin{aligned}
S_{t+\ell-1}\left(R_{t-1}\right) & <S_{t+\ell}\left(R_{\iota}\right)+S_{t+\ell-1}(p) / 2+S_{t+\ell-1}(q) / 2 \\
& <D(p, q)+\epsilon+S_{t+\ell-1}(p) / 2+S_{t+\ell-1}(q) / 2 .
\end{aligned}
$$

Furthermore, there is a continuum $R_{\imath-2}$ containing $p+q$ such that $R_{\mathrm{s} \rightarrow 2}$ is adjacent to $M-H_{t}$ and 


$$
\begin{aligned}
S_{t+t-2}\left(R_{t-2}\right)< & S_{t+\ell-1}\left(R_{t-1}\right)+S_{t+\varepsilon-2}(p) / 2+S_{t+\varepsilon-2}(q) / 2 \\
< & D(p, q)+\epsilon+S_{t+\ell-1}(p) / 2+S_{t+\ell-1}(q) / 2 \\
& +S_{t+\ell-2}(p) / 2+S_{t+\varepsilon-2}(q) / 2 .
\end{aligned}
$$

Continuing in this manner we find that there is a continuum $R_{0}$ containing $p+q$ such that $R_{0}$ is adjacent to $M-H_{t}$ and

$$
\begin{aligned}
S_{t}\left(R_{0}\right)< & D(p, q)+\epsilon \\
& +S_{t}(p) / 2+S_{t+1}(p) / 2+\cdots+S_{t+8-1}(p) / 2 \\
& +S_{t}(q) / 2+S_{t+1}(q) / 2+\cdots+S_{t+s-1}(q) / 2 .
\end{aligned}
$$

Since $K_{t}$ is not adjacent to $M-H_{t}, R_{0} \neq K_{t}$ and it follows from inequality (2) that

$$
S_{t}\left(K_{t}\right)+1 / 4^{n_{1}+n_{2}+\cdots+n_{t}} \leqq S_{t}\left(R_{0}\right) .
$$

It follows from inequality (5) that

$$
\begin{aligned}
D(p, q) \leqq & E_{t}(p, q)-S_{t}(p) / 2-S_{t+1}(p) / 2-\cdots \\
& -S_{t}(q) / 2-S_{t+1}(q) / 2-\cdots+1 /\left(3 \cdot 4^{n_{1}+n_{2}+\cdots+n_{t}}\right) .
\end{aligned}
$$

But inequalities (7), (6), and (8) imply that

$$
\begin{aligned}
1 / 4^{n_{1}+n_{2}}+\cdots+n_{t}< & \epsilon-S_{t+8}(p) / 2-S_{t+\ell+1}(p) / 2-\cdots \\
& -S_{t+\bullet}(q) / 2-S_{t+\imath+1}(q) / 2-\cdots \\
& +1 /\left(3 \cdot 4^{n_{1}+n_{2}+\cdots+n_{t}}\right) .
\end{aligned}
$$

Hence, if we take $\epsilon=2 /\left(3 \cdot 4^{n_{1}+n_{s}+\cdots+n_{t}}\right)$, we find that the supposition that there is a point $r$ between $p$ and $q$ which is not on $p q$ leads to a contradiction.

4. Examples and questions. Even though a continuous curve has a convex metric, this metric may be quite different from a Euclidean metric. For example, if $S$ is a horizontally based square plus its interior in the plane and $D(p, q)$ is defined to be the sum of the absolute values of the difference of the ordinates and the difference of the abscissas of $p$ and $q$, each point of $S$ lies on some segment between opposite vertices of $S$. It is of interest to get convex metrics that resemble Euclidean metrics.

Continuous curves each of whose segments is unique. If $I^{n}$ is a cube plus its interior in Euclidean $n$-space, its Euclidean metric causes each pair of its points to belong to a unique segment. Also, if $M$ is a continuous curve each of whose nondegenerate cyclic elements is topologically a cube of some dimension, $M$ has such a convex metric. Hence, each unicoherent plane continuous curve has such a metric. 
If $C$ is a cone whose base is a figure eight, $C$ may be regarded as the sum of two closed discs that are sewn together along a radius of each. This shows that $I^{2}$ is not the only continuous curve which is 2-dimensional at each of its points and has a convex metric under which all segments are unique.

Suppose $M$ is a continuous curve with a convex metric under which each pair of points belongs to a unique segment. Then for each point $p$ of $M$ there is a continuous transformation $F(m, t)$ ( $m$ element of $M$, $t$ element of $[0,1])$ of the cartesian product of $M$ and a straight line interval onto $M$ such that $F(m, 1)=m, F(m, 0)=p, F\left(F\left(m, t_{1}\right), t_{2}\right)$ $=F\left(m, t_{1}, t_{2}\right)$. By letting $t_{1}=0$ we find that the last equation implies that $F(p, t)=p$. We can define $F(m, t)$ as the point which divides the segment from $p$ to $m$ in the same ratio that $t$ divides the interval from 0 to 1 .

The interior of each sphere of $M$ is contractible to its center.

Questions. Does the existence of such a transformation $F(m, t)$ guarantee that $M$ can be assigned a convex metric under which segments are unique?

Not only would it be interesting to know a topological characterization of continuous curves which have convex metrics with only unique segments but it would also be interesting to know which continuous curves have the following type of convex metric. If two segments in Euclidean space intersect in more than one point, their sum is a segment. If an $n$-dimensional continuous curve has such a convex metric, is it necessarily topologically equivalent to an $n$-cube $I^{n}$ ?

The surface of a sphere has a convex metric under which nearby pairs of points belong to a unique segment. One might wonder if the local structure of a continuous curve determines whether or not it has such a convex metric-that is, does a continuous curve $M$ have such a metric if for each point $p$ of $M$ there is an open subset $N_{p}$ of $M$ containing $p$ such that $N_{p}$ is topologically equivalent to a set with such a metric?

Continuous curves with nonunique segments. The surface of a sphere $S^{n}$ in Euclidean $(n+1)$-space has the property that if $p$ is one of its points there is a point $q$ of it such that there is not a unique segment from $p$ to $q$. We know this because $S^{n}$ is not contractible-that is, it cannot be shrunk to a point [4]. Similarly, a continuous curve which is not unicoherent is not contractible [7] and therefore does not have a unique segment between each pair of its points.

Not each contractible continuous curve has a convex metric with only unique segments. Suppose that in the plane $J_{i}(i=1,2, \cdots)$ is a circle with radius $1 / i$ and center at $(1 / i, 0)$. If $C$ is a cone with 
$J_{1}+J_{2}+\cdots$ as a base, it is contractible but it does not have a convex metric with only unique segments because there is no contractible neighborhood not containing the vertex about the point $(0,0)$ of the base.

\section{REFERENCES}

1. R. H. Bing, Partitioning a set, Bull. Amer. Math. Soc. vol. 55 (1949) pp. 11011110.

2. Complementary domains of continuous curves, Fund. Math. vol. 36 (1949) pp. 306-318.

3. - Partitioning continuous curves, Bull. Amer. Math. Soc. vol. 58 (1952) pp. 536-556. 1948.

4. W. Hurewicz and H. Wallman, Dimension theory, Princeton University Press,

5. E. E. Moise, Grille decomposition and convexification theorems for compact metric locally connected continua, Bull. Amer. Math. Soc. vol. 55 (1949) pp. 1111-1121.

6. - , A note of correction, Proceedings of American Mathematical Society vol. 2 (1951) p. 838.

7. G. T. Whyburn, Analytic topology, Amer. Math. Soc. Colloquium Publications, vol. 28, New York, 1942.

THE UNIVERSITY OF Wisconsin 